Katarzyna Białczyk ${ }^{1 *(1)}$, Monika Schultz ${ }^{2 \star}$ Dorota Łuszkiewicz ${ }^{1}$, Jakub Lisiecki ${ }^{1}$, Robert Szafkowski ${ }^{1}$, Anna Kanigowska ${ }^{1}$

${ }^{1}$ Nicolaus Copernicus University in Torun, Collegium Medicum, Bydgoszcz, Poland

${ }^{2}$ Collegium Medicum Nicolaus Copernicus University, Ph student

*Monika Schultz and Katarzyna Białczyk have equally contributed to the present paper and should be considered to be the first authors

\title{
Abscopal effect in solid tumors. Potential mechanisms, application of radioimmunotherapy, and a review of exemplary clinical cases
}

\author{
Corresponding author: \\ Katarzyna Białczyk, Nicolaus \\ Copernicus University in Torun, \\ Collegium Medicum, Bydgoszcz, \\ Poland, \\ e-mail: katarzyna.bialczyk@cm.umk.pl \\ Medical Research Journal 2021: \\ Volume 6, Number 4, 334-341 \\ 10.5603/MRJ.a2021.0054 \\ Copyright (C) 2021 Via Medica \\ ISSN 2451-2591 \\ e-ISSN 2451-4101
}

\begin{abstract}
The abscopal effect, also known as extra-local, is a phenomenon in which local treatment of a neoplastic lesion causes regression of metastatic lesions located at a considerable distance from it.

The mechanism of this phenomenon is still not fully understood, however, there are some assumptions about its pathophysiology. In 2004, it was postulated for the first time that the immune system might be responsible for anti-cancer activities in this mechanism. This study aims to present the phenomenon of the abscopal effect, its probable mechanisms, to discuss the anti-tumor effect of radiotherapy combined with immunotherapy, and to present clinical cases.
\end{abstract}

Key words: abscopal effect, solid tumors, mechanisms, clinical cases

Med Res J 2021; 6 (4): 334-341

\section{Introduction}

The abscopal effect, also known as extra-local, is a phenomenon in which local treatment of a neoplastic lesion causes regression of metastatic lesions located at a considerable distance from it. R.H. Moles first introduced the term "abscopal effect" in 1953 in relation to the effects of ionizing radiation that were observed at some distance from the irradiated site but within the same organism [1]. The word abscopal comes from Latin, from the prefix "ab" meaning - apart and "scopos" - the purpose of the action. Topical radiotherapy has been shown in many case reports to induce an abscopal effect in various types of cancer, including in melanoma, esophageal adenocarcinoma, hepatocellular carcinoma, renal cell carcinoma, or lymphomas [2-5]. In the case of hematological neoplasms, such as lymphomas or leukemias, there is rather a pseudo-abscopal effect, which results from the circulation of lymphocytes in the irradiation field during its duration [6]. Due to the existing differences, this study deals with the abscopal effect occurring in the course of non-hematological neoplasms. The mechanism of this phenomenon is still not fully understood, however, there are some assumptions about its pathophysiology. In 2004, it was postulated for the first time that the immune system might be responsible for anti-cancer activities in this mechanism [7]. Since then, more and more data have appeared to support these assumptions. This study aims to present the phenomenon of the abscopal effect and its probable mechanisms; it will discuss the anti-tumor effect of radiotherapy combined with immunotherapy and present a few examples of clinical cases.

It should also be emphasized that the abscopal effect is a phenomenon separate from the so-called neighborhood effect, also known as the radiation, induced bystander effect (RIBE). The latter is based on the response of neoplastic cells that are close to the irradiated volume but not directly exposed to ionizing radiation [8]. The neighborhood effect is not the subject of this article.

\section{Mechanisms responsible for the abscopal effect}

There are several independent mechanisms responsible for the abscopal effect. Responses to radiation- 
-induced stress and inflammation play a key role. Local activation of intratumor or peritumor cells of the immune system can induce distal immune responses, as well as factors secreted by irradiated cells in the surrounding tissue, which can affect more distant cells. In all the cases, the in vivo abscopalous effects are mediated by cytokines released by activated T cells, which can move from irradiated to non-irradiated fields [9].

\section{Radiation-induced cytokine release}

Radiotherapy induces both local and systemic cytokine release. Cytokines play an important role in cell signaling during inflammation, mediate acute and late tissue responses, and possibly extraneous tumor regression. Irradiation and the formation of free oxygen radicals are accompanied by the activation of macrophages and the production of cytokines such as IL-1a, IL-1b, IL-6, TNF-alpha, TGF-b, or IFN type I [10, 11].

The radiation-induced cascade of type I and II interferons within the tumor plays a very important role in the anti-tumor immune response. It is necessary for the activation and function of dendritic cells and T lymphocytes (IFN $\beta$ ). Type I IFNs stimulate the cross-presentation of antigen by dendritic cells, enhance the maturation of T lymphocytes in the lymph nodes, and thus enhance the action of $\mathrm{T}$ lymphocytes against cancer cells. In turn, activated T lymphocytes and NK cells secrete type II interferons (IFN $\gamma$ ) increase the expression of MHC class I on tumor cells, ultimately resulting in their increased recognition by the immune system. Moreover, IFN $\gamma$ has been shown to modulate the tumor vasculature to improve T cell transport, and type I IFNs increase T cell recruitment by stimulating the secretion of chemokines [12]. Most importantly, the efficacy of radiotherapy in immunocompetent tumor-bearing mice was abolished in the absence of type I IFN signaling [13].

\section{Radiation-induced activation of the immune system}

The process of eliciting the anti-tumor immune effect induced by radiation therapy involves several steps including promoting the uptake of dying tumor cells by dendritic cells, cross-presentation of tumor-derived antigens, and activation of $\mathrm{T}$ cells.

As in the case of immune reactions to bacterial or viral antigens, the occurrence of the abscopal effect requires the activation of cells of the immune system against neoplastic antigens [12]. Local irradiation of a neoplastic tumor may lead to immunogenic cell death (ICD) [14], a type of apoptosis that leads to an increase in the immune system response and the release of many alarmin-like antigens called DAMP (damage-associated molecular patterns, e.g. calreticulin, ATP, HMGB1, IFN type I), which induce an anti-tumor response [15]. These antigens can be recognized and processed by antigen-presenting cells within the tumor, such as dendritic cells. The next step in inducing an immune response is reaching secondary lymphoid organs to present tumor antigens to $\mathrm{T}$ lymphocytes. For this purpose, dendritic cells migrate to the lymph nodes, where CD8 + T lymphocytes recognize tumor antigens presented to them in combination with MHC class I molecules [16]. As a result of the subsequent activation, cytotoxic T lymphocytes secrete cytokines that support or activate subsequent cells of the immune system or directly destroy neoplastic cells. Cytotoxic T cells circulate in the bloodstream and are therefore able to destroy any remaining cancer cells in distant parts of the body that have not been irradiated. Irradiation has been shown to increase the $T$ cell response to antigen presentation. This effect may be even more pronounced with stereotaxic radiotherapy [17-19]. It has been shown that an increase in tumor-specific cytotoxic $\mathrm{T}$ lymphocytes correlates with the occurrence of the abscopal effect in patients [20]. On the other hand, the abscopal effect is impossible to observe after an experimental reduction of the number of $\mathrm{T}$ lymphocytes in various animal models [21, 22].

\section{Other mechanisms}

Another mechanism responsible for the occurrence of the anti-tumor abscopal effect may depend on the function of the p53 gene, as presented in one of the publications. The authors showed that this effect was not observed in the absence of functional p53 or in mice in which it was pharmacologically inhibited [23]. It can be assumed that many other mechanisms are responsible for the abscopal effect, which are not yet fully understood, but which are the subject of intensive research.

\section{A combination of immunotherapy and radiation therapy to increase the anti-cancer effect}

The abscopal effect of ionizing radiation is often blocked by the immunosuppressive microenvironment inside the irradiated tumor, which prevents effective stimulation of $\mathrm{T}$ lymphocytes. During its growth, the tumor generates immune escape mechanisms that allow it to remain beyond the control of the immune system [24]. For example, tumor cells can reduce MHC class I expression or produce immunosuppressive cytokines. The overexpression of PD-L1 (and. Programmed death-ligand 1, a ligand for the cell death receptor) is also observed in the plasma membrane of neoplastic cells [25]. The PD-1 receptor (programmed death-1) together with the PD-L1 ligand create a specific checkpoint - a physiological mechanism designed to protect healthy cells of the body against self-destruction by host lymphocytes. In the case of neoplastic cells, 
this is a negative phenomenon, as it allows tumor cells to escape from the surveillance of the immune system. This explains why the abscopal effect is so rarely seen in patients receiving radiation alone. The use of immune checkpoint inhibitors $(\mathrm{ICl})$, i.e. antibodies that work synergistically with radiotherapy, allows for an abscopal effect. The combination of immunomodulatory drugs, such as ipilimumab [a monoclonal antibody that blocks the cytotoxic T lymphocyte-associated antigen 4 (CTLA-4)] and pembrolizumab (a monoclonal antibody directed against the PD-1 receptor), may partially reactivate the systemic anti-tumor immune responses induced after local radiation therapy of tumors [26].

In order to strengthen the immune response against metastatic changes in neoplasms of various origins, research has begun on combining radiotherapy with immunomodulating drugs. So far, many different substances have been tested for their ability to enhance the systemic anti-cancer effect. However, the optimal combination of radiation dose and fractionation with immunomodulating drugs is still the subject of intense research. Currently, the greatest hopes are related to CTLA-4 inhibitors and PD1/PD-L1 inhibitors.

\section{CTLA-4 inhibitors}

CTLA-4 is expressed on the surface of T lymphocytes activated as a result of contact with the antigen but is absent on T-lymphocytes. Therefore, CTLA-4 is expressed in lymphocytes showing the presence of CD28 [27]. CTLA-4 acts primarily as a molecule that inhibits the activation of T lymphocytes [28]; thus it is an element of the negative feedback loop of the immune response. Therefore, blocking CTLA-4 is considered a promising immunotherapeutic method to enhance the anti-tumor immune response, and a series of preclinical and clinical studies have shown the anti-tumor activity of CTLA-4 blockade in solid tumors, primarily in melanoma patients.

One of the articles by Postow et al. [20] showed a 30 -fold increase in the rate of antibodies to NYESO-1 caused by radiotherapy. NY-ESO-1 is an antigen expressed in $30-40 \%$ of patients with advanced melanoma. Patients with a high percentage of NY-ESO-1 antibodies have an increased likelihood of benefiting from ipilimumab treatment [29]. Thus, it has been proven that radiotherapy increases the effectiveness of ipilimumab [20]. This combination-treatment strategy has shown encouraging results in clinical trials and has been approved as a treatment for metastatic melanoma by the US Food and Drug Administration (FDA) [30].

In one retrospective study, Grimaldi et al. [31] documented promising results in patients with advanced melanoma treated with ipilimumab followed by radiotherapy. Of 21 patients, 11 (52\%) experienced an abscopal effect, including 9 who achieved a partial response and 2 who achieved stable disease. The over- all survival (OS) in patients with the abscopal effect was 22.4 months, compared with 8.3 months for patients who did not experience this effect. Similarly, in another retrospective analysis, Koller et al. [32] showed that in patients with advanced-stage melanoma who received ipilimumab in combination with radiotherapy, median OS and complete response rates increased significantly compared to those who did not receive such treatment. Nevertheless, more research is needed to combine radiotherapy with CTLA-4 blocking therapy.

\section{PD-1/PD-L1 inhibitors}

As with anti-CTLA-4 treatment, inhibition of the PD-1/PD-L1 immune checkpoint enhances the activation of $\mathrm{T}$ lymphocytes, thus facilitating cell-dependent anti-tumor immunity $[33,34]$. In one retrospective study of patients with metastatic melanoma who received PD-1 checkpoint inhibitors, Aboudaram et al. compared, among others, survival data and overall response rates in patients receiving concomitant or no radiation therapy. Of the 59 patients who received PD-1 blockade, 17 received palliative radiotherapy. The objective response rate, including the percentage of complete and partial responses, was significantly higher in the group receiving radiotherapy compared to the group not receiving radiotherapy after 10 months of follow-up, and one respondent had an abcopal effect. The 6-month disease-free survival and OS were slightly higher in the group of patients undergoing radiotherapy compared to the group not receiving radiotherapy [35]. Among other studies conducted so far, it is also worth quoting Ahmed's work from 2016 [36]. In this study, 26 patients with brain metastases received radiation therapy in combination with nivolumab, which resulted in increased survival (OS) compared to standard therapy.

While there are many encouraging reports on the combination of radiotherapy and anti-PD-1/PD-L1 antibodies, the frequency of the abcopal effects is still undefined. The key seems to be to identify those patients who are most likely to respond to the treatment, therefore there is a need for additional or ongoing studies that will determine characteristics of such patients.

\section{Clinical cases}

\section{Hepatocellular carcinoma}

Nakanishi et al. [4] presented the case of a 79-yearold patient diagnosed with multifocal hepatocellular carcinoma (HCC), whose disease regressed after irradiation of one of the lesions. The man was admitted for the treatment of many intrahepatic HCC tumors, diagnosed on the basis of computed tomography (CT) and angio-CT, and elevated levels of AFP and PIVKA-II in the blood serum without histopathological confirma- 
tion. The treatment consisted of selective transcatheter embolization of the artery and external beam radiation therapy (EBRT) up to a total dose of 48 Gy aimed at the dominant lesion in the right lobe of the liver. The remaining $\mathrm{HCC}$ changes were left untreated. After 5 months, both the treated and untreated lesions regressed, which was visualized in CT and angio-CT.

Another case of a 63-year-old male with HCC located in the right lobe of the liver was reported by Okuma et al. [37]. The patient underwent dilated right-sided lobectomy of the liver. The diagnosis of HCC was confirmed by histopathological examination. Eighteen months after surgery, the patient developed a single metastasis to the right lung and a single metastasis in the mediastinal lymph node. The patient underwent EBRT for a changed node in the mediastinum to relieve symptoms such as coughing and moderate hemoptysis. The total dose was $60.75 \mathrm{~Gy}$ and the fractional dose was 2.25 Gy. The patient did not receive any other treatment. The CT scan performed after radiotherapy showed a reduction in the lesion in the mediastinal lymph node, as well as a reduction in the size of the metastatic lesion in the lung that was outside the irradiation field, and a significant decrease in the level of AFP in the blood serum. During the 10-year follow-up, no chest recurrence was observed.

\section{Merkel cell carcinoma}

The only case of an abscopal effect in Merkel cell carcinoma has been reported by Cotter et al., and it concerned a 70-year-old patient with a primary tumor on the right lower leg [38]. One month after resection of the primary lesion and irradiation of the right shin area, multiple skin metastases on the right lower limb appeared. Eleven of the resulting changes in the upper part of the lower leg were irradiated using high-dose brachytherapy up to a total dose of 12 Gy in 2 equal fractions. Two lesions located in the lateral ankle of the right leg and the plantar part of the foot remained non-irradiated. All skin lesions, both irradiated and non-irradiated, completely disappeared within a few weeks after the end of treatment. During the 25-month follow-up, the patient did not develop any skin lesions in the above-mentioned locations.

\section{Lung adenocarcinoma}

Another case concerns a 78-year-old patient who was diagnosed with adenocarcinoma affecting both lungs [39]. There was a locally advanced tumor in the upper lobe of the left lung and a synchronous tumor in the lower lobe of the right lung. Both changes were verified by biopsy and were adenocarcinomas. The lesion in the left lung was treated with radiotherapy up to a total dose of $60 \mathrm{~Gy}$ in 30 fractions with simultaneous chemotherapy with carboplatin and paclitaxel. After 5 weeks of treatment, there was a complete response. The patient then received a single 26 Gy fraction using stereotactic ablative radiation therapy (SABR) alternately in the right lung. The response was assessed by a positron emission tomography/CT (PET-CT) scan. The study performed 14 days after SABR showed an increased metabolism in the lesion located in the lower lobe of the right lung and the appearance of a new metastasis in the right adrenal gland. In the examination performed on day 70 after SABR, a similar result was obtained with an additional focus on metastasis in the right humerus. The patient was offered chemotherapy, which she refused. Subsequent PET-CT studies showed a decrease in metabolic activity in the lesion in the right lung and the area of metastatic lesions in the adrenal gland and humerus. Moreover, the study performed 12 months after the end of SABR showed a complete response in all locations.

Golden et al. [40] presented the first case concerning the abscopal effect in a treatment-resistant lung cancer treated with radiotherapy in combination with ipilimumab. The description concerned a 64-year-old patient with metastatic adenocarcinoma of the lung. After multiple lines of chemotherapy and prior lung irradiation, the disease progressed. New metastatic changes appeared in the liver, in the sacrum, and the peraortic lymph nodes, and later also in the thoracolumbar spine, pelvic bones, and the right humerus. The patient was given ipilimumab and radiotherapy to one of the metastatic lesions in the liver to induce an abscopal effect. The technique of intensity-modulated radiation therapy was used up to a total dose of 30 Gy in 5 fractions administered over 2 weeks. The first course of ipilimumab was introduced during radiotherapy, followed by 3 more cycles. After radiotherapy in combination with ipilimumab, imaging tests were performed, which showed a significant regression of the lesion in the liver after 2.5 months. PET-CT examination performed 5 months after radiotherapy showed a reduction in changes in the liver, bones, and lung. Histopathological evaluation of the persistent supraclavicular lymph node showed a high percentage of CD8+ cells. The normalization of tumor markers was also observed. The patient remained without any signs of disease for one year of follow-up.

\section{Esophageal adenocarcinoma}

The case described by Bruton and Truong [3] concerned a 74-year-old patient with esophageal adenocarcinoma with metastases to the periophageal lymph nodes and the abdominal lymph nodes at the level of the right renal vessels [3]. The patient received palliative radiotherapy to the area of the primary tumor in the esophagus and regional lymph nodes up to a total 
dose of 30 Gy in 10 fractions. The lymph nodes in the abdominal cavity were outside the irradiation area. A CT scan 1.5 months after the end of radiotherapy showed a persistent thickening in the lower esophagus and a reduction in the volume of metastatic lymph nodes. The patient refused the offer of chemotherapy but remained under surveillance. A subsequent CT scan after another 5 months of follow-up showed a complete response within the irradiated area including the primary tumor and regional lymph nodes, and a stable metastatic lymph node at the right renal vein. After another 8 months, a PET scan was performed which showed little residual activity in the distal part of the esophagus and no activity in the irradiated and non-irradiated lymph nodes.

\section{Renal cell carcinoma}

Wersäll et al. [5] described 4 cases of the abscopal effect in the course of renal cell carcinoma. The first one concerned an 83-year-old patient in poor general condition diagnosed with right kidney cancer with multiple metastases to the lymph nodes in the hilum area and the lungs. Due to the general condition of the patient, she was not subjected to surgical treatment, but it was decided to treat the primary lesion in the right kidney (6 $\mathrm{cm}$ in diameter) with stereotaxic radiotherapy (8 Gy in 4 fractions at 3 fractions per week and heterogeneous dose distribution). The CT examination performed after 2 years of follow-up showed almost complete regression in the lymph nodes in the hilum area and complete regression of metastatic changes in the lungs. The primary irradiated lesion in the right kidney remained unchanged $(5.5 \mathrm{~cm}$ in diameter).

The second patient was a 64-year-old patient with renal cell carcinoma of the right kidney, $7 \mathrm{~cm}$ in diameter, who underwent primary nephrectomy. Two months later, the patient developed multiple bilateral lung metastases and was treated with interferon together with interleukin 2 (IL-2). One month after starting therapy, it was discontinued due to side effects. Due to the patient's interest in stereotaxic radiotherapy, it was decided to start this form of treatment aimed at metastatic lesions in the lungs. Irradiation was administered in 5 shifts in 2 sessions separated by an interval of 2 months. The 5-month CT scan showed complete or partial regression of irradiated lesions and complete regression of one and partial regression of five non-irradiated metastatic lesions. It was decided to use stereotaxic radiotherapy directed at lesions showing partial regression that had not been previously irradiated. Six months before the last irradiation, the patient was also treated with a low dose of thalidomide (50 mg/day). Six months after the end of radiotherapy, a CT scan was performed, which showed regression of the treated lesions and no ap- pearance of new lesions. Four years after the end of treatment, the patient did not relapse.

The third described case concerned a 69-year-old man diagnosed with renal cell carcinoma of the right kidney, $10 \mathrm{~cm}$ in diameter, with metastases to the lungs. The patient underwent primary nephrectomy, after which he received stereotaxic radiotherapy for the two largest lung metastases up to a total dose of 30 Gy (2 fractions of 15 Gy each). A CT scan performed 3 months after the end of radiotherapy showed complete regression of changes subjected to irradiation and partial regression of other changes in the lungs.

The last described patient, aged 55, was diagnosed with renal cell carcinoma of the right kidney with extensive involvement of the lymph nodes. A nephrectomy was attempted, but it was unsuccessful due to the extensive changes in the intraoperative evaluation. The primary lesion was irradiated with stereotaxic radiotherapy ( 8 Gy in 4 fractions, 3 fractions a week). Two months after the end of treatment, the patient developed a new suspicious focal lesion in the left lung with a diameter of $5 \mathrm{~mm}$, while the remaining lesions were stable on the CT examination. The PET-CT scan performed 5 months after the end of radiotherapy did not show any activity in any of the lesions, and the control examinations performed after 6 and 9 months did not show the previously described lesion in the lung and showed stabilization of the remaining lesions.

The occurrence of the abscopal effect in the course of kidney cancer was also observed by Ishiyama et al. [41]. The case report concerned a 61-year-old patient who underwent a left-sided nephrectomy for renal cell carcinoma with metastases to the adrenal gland. Other metastatic lesions were also imaged in both lungs, mediastinum, left hip socket, vertebrae of the thoracic spine, and in the brain. The patient received treatment including radiosurgery (RS) for metastatic lesions in the brain at a dose of $18 \mathrm{~Gy}$ and stereotaxic radiotherapy for changes in the spine up to a total dose of 40 Gy given in 5 fractions. The patient did not receive any systemic therapy. The first follow-up CT scan one month after the end of treatment showed a slight regression of changes in the non-irradiated lungs and mediastinum. These changes gradually decreased in subsequent studies and were slightly noticeable after 4 months. In the meantime, the patient developed new brain metastases one month and three months after stopping treatment, however, previously irradiated lesions were well controlled. New metastatic lesions also underwent RS. After 2.5 years, all previously present changes remained stable and no new metastatic lesions appeared. The mixed response observed in this case, including simultaneous regression of changes and disease progression, led the author of the publication to suggest that the occurrence of the abscopal effect may depend 
on the organ involved and is probably blocked by the blood-brain barrier.

\section{Melanoma}

In 2012, Postow et al. [20] noticed a correlation between the immune system and the abscopalous effect in a 33-year-old female patient diagnosed with metastatic malignant melanoma expressing NY-ESO-1. The primary lesion was located on the skin of the upper back. The lesion was excised with an appropriate margin of healthy tissues and sentinel node biopsy was performed in the left feed. After a few years, the disease progressed and a metastatic focus appeared in the paraspinal pleura; there were also changes in the hilum of the right lung and the spleen. The patient started treatment with ipilimumab as a maintenance treatment. In order to relieve pain in the right part of the back caused by paraspinal metastatic lesions, it was decided to implement palliative radiotherapy in the area of the above-mentioned focus. The intensity-modulated radiation therapy technique was applied to a total dose of $28.5 \mathrm{~Gy}$ in 3 fractions. A CT scan performed one month after the end of radiotherapy showed no response in the irradiated lesion, therefore, it was decided to administer another course of ipilimumab. Four months later, the previously irradiated paraspinal lesion regressed completely, and the non-irradiated lesions located in the hilum of the right lung and the spleen also regressed. In the CT scan performed 10 months after the end of radiotherapy, the disease picture remained stable.

In the same year, another case of the abscopal effect was described during ipilimumab therapy in combination with radiotherapy [42]. It concerned a 57-year-old man diagnosed with malignant melanoma with metastases, including to the liver. The patient was treated with two cycles of ipilimumab, followed by irradiation of 2 out of 8 metastatic changes in the liver with stereotaxic radiotherapy and another two additional cycles of ipilimumab. A PET-CT scan performed after 4 months showed that there was a complete response in all metastatic lesions, including non-irradiated liver lesions and the lesion located in the subcutaneous tissue of the armpit, which completely disappeared after 2 months.

Another case of the abscopal effect in melanoma was presented by Stamell et al. [43]. In a 67-year-old patient, the disease comprised a primary lesion located on the scalp and numerous satellite lesions. Despite chemotherapy, the primary lesion progressed and was then irradiated with a $2 \mathrm{~cm}$ margin. The total dose of 24 Gy was applied in 3 fractions, using an electron beam with an energy of $6 \mathrm{MeV}$. After 8 months of observation, the primary lesion became flat, showed little pigmentation, but without clinical signs of the residual tumor mass. Neighboring satellite outbreaks not covered by the irradiation area also completely regressed. The patient has relapsed with metastases to the lymph nodes in the neck and the brain. The lesions in the neck lymph nodes were removed during surgery, while the brain metastases were treated with RS and immunotherapy with ipilimumab. This treatment showed an increase in antibody titers against melanoma-associated A3 antigen (MAGE-A3), indicating a systemic anti-tumor immune response. The patient remained without relapse 7 years after the end of treatment.

\section{Giant cell tumor of bone}

An atypical case of the abscopal effect in a 26-yearold patient with a giant cell tumor of the left ulna was observed at the Lower Silesian Oncology Center in Wrocław [44]. In the patient, dissemination of the disease was ruled out and the tumor was removed together with resection of $1 / 3$ of the distal ulna. The postoperative histopathological examination revealed signs of potential malignancy and a tendency to recurrence. Approximately 2 years after the surgery, the patient's disease recurred in the previously operated area. Additionally, imaging studies revealed numerous metastases in both lungs. The patient was enrolled in denosumab treatment in a clinical trial, from which he was withdrawn after some time due to lung progression. Then chemotherapy was administered, during which the disease progressed further. It was decided to change the chemotherapy regimen to ifosfamide and to apply palliative radiotherapy to the area of the forearm tumor up to a dose of 30 Gy in 10 fractions, after which an improvement was achieved. Palliative ifosfamide chemotherapy was also continued. During the therapy, local improvement and stabilization of the disease in the lungs were observed, but after some time the lesions progressed both in the lungs and in the limb. The patient was qualified for renewed radiotherapy to the recurrence area up to a dose of 30 Gy in 10 fractions. After the treatment was completed, subsequent imaging studies showed a gradual reduction in changes in the lungs. After 1.5 years of follow-up, the disease stabilized, with no signs of progression and no symptoms related to the disease.

\section{Summary and future lines of research to increase the incidence of abscopal effects in radioimmunotherapy}

To date, many retrospective clinical trials of combination therapy with radiotherapy and checkpoint inhibitors have been conducted. However, before such treatment of metastatic neoplastic disease is considered standard, it is necessary to conduct thorough 
prospective randomized studies. The subject of the research should also include issues such as optimal dosing regimens of checkpoint inhibitors, regimens of combining radiotherapy with immunomodulating drugs, and optimal dosing and fractionation regimens of radiotherapy in the context of radioimmunotherapy, in order to induce effective abscopal effects. It seems that the wider use of stereotaxic radiotherapy and RS, which are becoming more and more common in the treatment of neoplastic lesions of various locations, may increase the chances of an abscopal effect. While the combination of immunotherapy and radiation therapy has shown promising results in the treatment of many solid tumors, not all patients experienced the abscopal effect. Therefore, it is also necessary to identify effective biomarkers that could predict abscopal effects in patients receiving combination radioimmunotherapy regimens. In addition, validated biomarkers would help select the right patients, identify optimal therapeutic strategies, and predict treatment response.

\section{Conflict of interest: None.}

Funding: None.

\section{References}

1. Mole $\mathrm{RH}$. Whole body irradiation; radiobiology or medicine? Br J Radiol 1953: 26(305): 234-241, doi: 10.1259/0007-1285-26-305-234, indexed in Pubmed: 13042090.

2. Kingsley DPE. An interesting case of possible abscopal effect in malignant melanoma. Br J Radiol. 1975; 48(574): 863-866, doi 10.1259/0007-1285-48-574-863, indexed in Pubmed: 811297

3. Bruton Joe M, Truong PT. Abscopal effect after palliative radiation therapy for metastatic adenocarcinoma of the esophagus. $\mathrm{Cu}$ reus. 2018; 10(8): e3089, doi: 10.7759/cureus.3089, indexed in Pubmed: 30333943.

4. Nakanishi M, Chuma M, Hige S, et al. Abscopal effect on hepatocellular carcinoma. Am J Gastroenterol. 2008; 103(5): 1320-1321, doi 10.1111/j.1572-0241.2007.01782 13.x, indexed in Pubmed: 18477367

5. Wersäll PJ, Blomgren $H$, Pisa $P$, et al. Regression of non-irradiated metastases after extracranial stereotactic radiotherapy in metastatic renal cell carcinoma. Acta Oncol. 2006; 45(4): 493-497, doi 10.1080/02841860600604611, indexed in Pubmed: 16760190.

6. Kaminski JM, Shinohara E, Summers JB, et al. The controversial abscopal effect. Cancer Treat Rev. 2005; 31(3): 159-172, doi: 10.1016/j. ctrv2005.03.004 indexed in Pubmed: 15923088.

7. Demaria $\mathrm{S}, \mathrm{Ng} \mathrm{B}$, Devitt $\mathrm{ML}$, et al. lonizing radiation inhibition of distant untreated tumors (abscopal effect) is immune mediated. Int J Radiat Oncol Biol Phys. 2004; 58(3): 862-870, doi: 10.1016/j. ijrobp.2003.09.012, indexed in Pubmed: 14967443

8. Goldberg Z, Lehnert BE. Radiation-induced effects in unirradiated cells: a review and implications in cancer. Int J Oncol. 2002; 21(2) 337-349, indexed in Pubmed: 12118330

9. Sprung CN, Ivashkevich A, Forrester $\mathrm{HB}$, et al. Oxidative DNA damage caused by inflammation may link to stress-induced non-targeted effects. Cancer Lett. 2015; 356(1): 72-81, doi: 10.1016/j. canlet.2013.09.008, indexed in Pubmed: 24041866

10. Hallahan DE, Spriggs DR, Beckett MA, et al. Increased tumor necrosis factor alpha mRNA after cellular exposure to ionizing radiation. Proc Natl Acad Sci U S A. 1989; 86(24): 10104-10107, doi: 10.1073/pnas.86.24.10104, indexed in Pubmed: 2602359

11. Calveley VL, Khan MA, Yeung IWT, et al. Partial volume rat lung irradiation: temporal fluctuations of in-field and out-of-field DNA damage and inflammatory cytokines following irradiation. Int J Radiat Biol. 2005; 81(12): 887-899, doi: 10.1080/09553000600568002, indexed in Pubmed: 16524844

12. Brix $\mathrm{N}$, Tiefenthaller $\mathrm{A}$, Anders $\mathrm{H}$, et al. Abscopal, immunological effects of radiotherapy: Narrowing the gap between clinical and preclinical experiences. Immunol Rev. 2017; 280(1): 249-279, doi: 10.1111/imr.12573, indexed in Pubmed: 29027221.

13. Burnette $\mathrm{BC}$, Liang $\mathrm{H}$, Lee $\mathrm{Y}$, et al. The efficacy of radiotherapy relies upon induction of type i interferon-dependent innate and adaptive immunity. Cancer Res. 2011; 71(7): 2488-2496, doi: 10.1158/00085472. CAN-10-2820, indexed in Pubmed: 21300764.

14. Bezu L, Gomes-de-Silva LC, Dewitte H, et al. Combinatorial strategies for the induction of immunogenic cell death. Front Immunol. 2015; 6: 187, doi: 10.3389/fimmu.2015.00187, indexed in Pubmed: 25964783.

15. Garg AD, Dudek AM, Agostinis P. Cancer immunogenicity, danger signals, and DAMPs: what, when, and how? Biofactors. 2013; 39(4): 355-367, doi: 10.1002/biof.1125, indexed in Pubmed: 23900966.

16. Cummings RJ, Gerber SA, Judge JL, et al. Exposure to ionizing radiation induces the migration of cutaneous dendritic cells by a CCR7-dependent mechanism. J Immunol. 2012; 189(9): 4247-4257, doi: 10.4049/jimmunol.1201371, indexed in Pubmed: 23002435.

17. Sharabi AB, Nirschl CJ, Kochel CM, et al. Stereotactic radiation therapy augments antigen-specific $\mathrm{PD}-1$-mediated antitumor immune responses via cross-presentation of tumor antigen. Cancer Immunol Res. 2015; 3(4): 345-355, doi: 10.1158/2326-6066.CIR-14-0196, indexed in Pubmed: 25527358

18. Lee $Y$, Auh SL, Wang Y, et al. Therapeutic effects of ablative radiation on local tumor require CD8+ T cells: changing strategies for cancer treatment. Blood. 2009; 114(3): 589-595, doi: 10.1182/blood-2009-02-206870, indexed in Pubmed: 19349616.

19. Sharabi AB, Lim M, DeWeese TL, et al. Radiation and checkpoint blockade immunotherapy: radiosensitisation and potential mechanisms of synergy. Lancet Oncol. 2015; 16(13): e498-e509, doi: 10.1016/S14702045(15)00007-8, indexed in Pubmed: 26433823.

20. Postow MA, Callahan MK, Barker CA, et al. Immunologic correlates of the abscopal effect in a patient with melanoma. $\mathrm{N}$ Engl $\mathrm{J}$ Med. 2012; 366(10): 925-931, doi: 10.1056/NEJMoa1112824, indexed in Pubmed: 22397654

21. Rodriguez-Ruiz ME, Rodriguez I, Garasa S, et al. Abscopal effects of radiotherapy are enhanced by combined immunostimulatory mAbs and are dependent on CD8 t cells and crosspriming. Cancer Res. 2016; 76(20): 5994-6005, doi: 10.1158/0008-5472.CAN-16-0549, indexed in Pubmed: 27550452

22. Vanpouille-Box C, Diamond JM, Pilones KA, et al. TGF $\beta$ is a master regulator of radiation therapy-induced antitumor immunity. Cancer Res. 2015; 75(11): 2232-2242, doi: 10.1158/0008-5472.CAN-14-3511, indexed in Pubmed: 25858148

23. Camphausen K, Moses MA, Ménard C, et al. Radiation abscopal antitumor effect is mediated through p53. Cancer Res. 2003; 63(8): 1990-1993, indexed in Pubmed: 12702593

24. Lumniczky K, Sáfrány G. The impact of radiation therapy on the antitumor immunity: local effects and systemic consequences. Cancer Lett. 2015; 356(1): 114-125, doi: 10.1016/j.canlet.2013.08.024, indexed in Pubmed: 23994343

25. Parry RV Chemnitz JM, Frauwirth KA, et al. CTLA-4 and PD-1 receptors inhibit T-cell activation by distinct mechanisms. Mol Cell Biol. 2005; 25(21): 9543-9553, doi: 10.1128/MCB.25.21.9543-9553.2005, indexed in Pubmed: 16227604

26. Twyman-Saint Victor C, Rech AJ, Maity A, et al. Radiation and dual checkpoint blockade activate non-redundant immune mechanisms in cancer. Nature. 2015; 520(7547): 373-377, doi: 10.1038/nature14292, indexed in Pubmed: 25754329.

27. Lindsten T, Lee KP, Harris ES, et al. Characterization of CTLA-4 structure and expression on human T cells. J Immunol. 1993; 151(7): 3489-3499, indexed in Pubmed: 8397258.

28. Vandenborre K, Van Gool SW, Kasran A et al. Interaction of CTLA-4 (CD152) with CD80 or CD86 inhibits human T-cell activation. Immunology. 1999; 98(3): 413-421, doi: 10.1046/j.1365-2567.1999.00888.x, indexed in Pubmed: 10583602

29. Yuan J, Adamow M, Ginsberg BA, et al. Integrated NY-ESO-1 antibody and CD8 + T-cell responses correlate with clinical benefit in advanced melanoma patients treated with ipilimumab. Proc Natl Acad Sci U S A. 2011; 108(40): 16723-16728, doi: 10.1073/pnas.1110814108, indexed in Pubmed: 21933959

30. Hodi FS, O'Day SJ, McDermott DF, et al. Improved survival with ipilimumab in patients with metastatic melanoma. N Engl J Med. 2010; 363(8): 711-723, doi: 10.1056/NEJMoa1003466, indexed in Pubmed: 20525992 
31. Grimaldi AM, Simeone E, Giannarelli D, et al. Abscopal effects of radiotherapy on advanced melanoma patients who progressed after ipilimumab immunotherapy. Oncoimmunology. 2014; 3: e28780, doi: 10.4161/onci.28780, indexed in Pubmed: 25083318.

32. Koller KM, Mackley HB, Liu J, et al. Improved survival and complete response rates in patients with advanced melanoma treated with concurrent ipilimumab and radiotherapy versus ipilimumab alone. Cance Biol Ther. 2017; 18(1): 36-42, doi: 10.1080/15384047.2016.1264543, indexed in Pubmed: 27905824.

33. Zitvogel L, Kroemer G. Targeting PD-1/PD-L1 interactions for cancer immunotherapy. Oncoimmunology. 2012: 1(8): 1223-1225, doi: 10.4161/onci.21335, indexed in Pubmed: 23243584.

34. Mahoney KM, Freeman GJ, McDermott DF. The next immune-checkpoint inhibitors: PD-1/PD-L1 blockade in melanoma. Clin Ther. 2015; 37(4): 764-782, doi: 10.1016/j.clinthera.2015.02.018, indexed in Pubmed: 25823918.

35. Aboudaram A, Modesto A, Chaltiel L, et al. Concurrent radiotherapy for patients with metastatic melanoma and receiving anti-programmed-death 1 therapy: a safe and effective combination. Melanoma Res. 2017; 27(5): 485-491, doi: 10.1097/CMR.0000000000000386, indexed in Pubmed: 28858075.

36. Ahmed KA, Stallworth DG, Kim Y, et al. Clinical outcomes of melanoma brain metastases treated with stereotactic radiation and anti-PD-1 therapy. Ann Oncol. 2016; 27(3): 434-441, doi: 10.1093/annonc/mdv622 indexed in Pubmed: 26712903.

37. Okuma K, Yamashita H, Niibe Y, et al. Abscopal effect of radiation on lung metastases of hepatocellular carcinoma: a case report. J Med Case Rep. 2011; 5: 111, doi: 10.1186/1752-1947-5-111, indexed in Pubmed: 21418591.
38. Cotter SE, Dunn GP, Collins KM, et al. Abscopal effect in a patient with metastatic Merkel cell carcinoma following radiation therapy: potential role of induced antitumor immunity. Arch Dermatol. 2011; 147(7): 870-872, doi: 10.1001/archdermatol.2011.176, indexed in Pubmed: 21768497

39. Siva S, Callahan J, MacManus MP, et al. Abscopal [corrected] effects after conventional and stereotactic lung irradiation of non-small-cell lung cancer. J Thorac Oncol. 2013; 8(8): e71-e72, doi: 10.1097/JTO .0b013e318292c55a, indexed in Pubmed: 23857404.

40. Golden EB, Demaria S, Schiff PB, et al. An abscopal response to radiation and ipilimumab in a patient with metastatic non-small cell lung cancer. Cancer Immunol Res. 2013; 1(6): 365-372, doi: 10.1158/23266066.CIR-13-0115, indexed in Pubmed: 24563870.

41. Ishiyama $\mathrm{H}$, Teh BS, Ren $\mathrm{H}$, et al. Spontaneous regression of thoracic metastases while progression of brain metastases after stereotactic radiosurgery and stereotactic body radiotherapy for metastatic renal cell carcinoma: abscopal effect prevented by the blood-brain barrier? Clin Genitourin Cancer. 2012; 10(3): 196-198, doi: 10.1016/j. clgc.2012.01.004, indexed in Pubmed: 22409865.

42. Hiniker SM, Chen DS, Knox SJ. Abscopal effect in a patient with melanoma. N Engl J Med. 2012; 366(21): 2035-2036, doi: 10.1056/NEJMc1203984, indexed in Pubmed: 22621637.

43. Stamell EF, Wolchok JD, Gnjatic S, et al. The abscopal effect associated with a systemic anti-melanoma immune response. Int J Radiat Oncol Biol Phys. 2013; 85(2): 293-295, doi: 10.1016/j.jrobp.2012.03.017, indexed in Pubmed: 22560555.

44. Gabalewicz K, Filipczyk-Cisarż E. Giant-cell tumour of bone with lung metastases - case report. Oncol Clin Pract. 2017; 13(4): 169-171, doi: 10.5603/OCP.2017.0020 